\title{
NOTA SOBRE LA SIMILITUD Y LOS COLORES*
}

ADOLFO GARCía DÍAZ Univeraidad de Zulia

Las siguientes notas sólo adquirirán pleno sentido en el horizonte de una teoría más amplia todavía incompleta, si bien ciertos temas serán tratados independientemente de ella, a fin de ofrecer una idea más precisa de nuestra posición ante los problemas que suscitan. La principal pretensión de estas reflexiones consiste en dar razón de la similitud de los colores, así como de la identidad e individuación de los mismos. En relación a lo primero, deben mucho a las investigaciones, en proceso de desarrollo, del profesor José A. Robles, ${ }^{1}$ quien hasta ahora ha efectuado la importante tarea de desbrozar el camino de quienes se sientan atraídos por la búsqueda de soluciones a los problemas que presenta aclarar qué sea la semejanza entre colores; problemas erizados de dificultades con las que se ha tropezado varias veces a lo largo de la historia de la filosofía.

Detrás de las penetrantes críticas del profesor Robles se adivina una solución. No creemos que coincida con la nuestra. Pero, puesto que nuestra intención ahora no es señalar quién está errado en qué o cómo, sino que ambos estamos particularmente interesados en la cuestión, pasaré a exponer mis razones directamente.

Para ejemplificar, en términos muy generales, el marco en que surgen las más señaladas dificultades acerca de la

- Conferencia dada en el Instituto de Investigaciones Filosóficas de la UNAM, el 23 de julio de 1979. Su publicación está dedicada a la maestra Margarita Ponce, secretaria académica de dicho Instituto, como testimonio de amistad.

1 Véase J. A. Robles, "Cualidades (simples) y semejanza”, Crítica, 26, vol. IX, 1977; "Cualidades (simples) y semejanza II" (en proceso). 
noción de similitud, citaré las que se presentan al tratar de concebir la semejanza de los colores, si es que éstos se nos dan como simples, al modo en que se ha concebido la identidad; es decir, las dificultades de tratar de sostener que, cuando decimos que este color es similar a este otro, habría que añadir: similar en tal o en cual, a la manera en que tal vez se debiera añadir, en el caso de la identidad entre $a$ y $b$, un término general: $a$ es el mismo $\mathrm{F}$ que $b^{2}$

Antes de proseguir haremos una advertencia previa. En lo que sigue no tomaremos en cuenta ni el brillo ni la saturación de los colores, y a los llamados matices los consideraremos simplemente como colores diferentes. Excluiremos también toda consideración del color en términos teóricocientíficos, en el sentido de que el color no fuera sino un tren de ondas de cierto tipo, o un patrón peculiar de estímulo retinal; ciertas distribuciones en la corteza visual, o determinadas respuestas de un observador ante ciertos estímulos. Se ha hecho la jocosa observación de que un científico, que supiera todo lo que hay que saber del color azul, continuaría viendo el cielo como lo veía en su infancia. El azul que ahora ve no se le ha transformado en una telaraña de unidades teóricas elementales. ${ }^{8}$ Tomaremos, pues, a los colores tal como se nos dan de inmediato en un conocimiento directo.

Para comenzar, llevemos a cabo un Gedankenexperiment. Supóngase que conocemos directamente un color, entendido tal conocimiento como fuera de toda teoría y sin ningún ropaje lingüístico. De inmediato nos surgen dudas acerca del sentido que hay que dar a esta suposición. ¿Se trata del conocimiento de un único color o del de una parte de un único color? Lo último es entendible; pero ciertamente nos veríamos en dificultades para entender lo que significaría cono-

2 Véase P. T. Geach, Reference and Generality, Cornell University Press, 1970 , p. 39.

3 R. M. Yust, "Some Philosophical Problems of Perception", en Carterette y Friedman (eds.), Handbook of Perception, vol. L, Academic Press, New York, 1974, p. 38. 
cer un único color y ninguno más. Para abreviar, reduzcamos la cuestión al caso de que nunca se nos diera más que un solo color. Basta un poco de reflexión para percatarnos que ello no es factible. Imposibilitados para saber cuál color no es, tampoco podríamos decir cuál es; más aún, no sabríamos ni siquiera si es color. Esto se verá más claramente a lo largo de lo que sigue: pero, por lo pronto, modifiquemos nuestro experimento. Supongamos que conocemos directamente dos colores. De nuevo se nos presentan dudas. ¿Qué ocurre si, en un momento dado, no estamos seguros de si conocemos uno o dos colores? ¿Se nos dan directamente, entonces, dos o sólo uno? Para evitar más digresiones, convengamos en que conocemos dos colores indudablemente y que la diferencia entre ellos es la mínima suficiente para que se nos den dos y no uno. Si se piensa que hubiera, además de nuestros colores $\mathrm{A}$ y $\mathrm{B}$, un tercer color $C$, cuya diferencia con $A$, por ejemplo, pudiera ser aún menor que la de $B$ respecto a $A$, entonces simplemente haremos un intercambio $y$ tomaremos a $C$ en lugar de $B$, si bien, dado que la discriminación efectiva entre colores tiene un límite - así no sea muy definido-, este intercambio deberá tener también un límite.

Cabe preguntarse ahora si estos dos colores son similares, pues parece que lo fueran por diferir mínimamente, esto es, lo suficiente para ser distinguidos sin vacilación. Sin embargo, esto no es aceptable. Lo que tenemos es precisamente una diferencia, por mínima que sea, no una similitud. Tal vez se nos ocurriera pensar, llevados por la tradición filosófica, que algo deben tener en común y algo de diferente para ser similares. Pero nada pueden tener de común y propio; difiriendo mínimamente, difieren totalmente, pues lo mínimo de su diferir sólo mienta un mínimo indispensable para que se nos presenten como dos al conocerlos directamente. Se podría objetar que, por lo menos, algo deben tener en común para ser colores y no otra cosa. Presentar, por ejemplo, una misma relación, en cuanto objetos inten- 
cionales de la visión, o ser ideas producidas por la vista, según lo entiende Locke, ${ }^{\star}$ o algo parecido.

$\mathrm{Al}$ respecto debemos señalar que, cuando hemos dicho que A y $\mathrm{B}$ son colores, no hemos aludido a nada común en ellos, sino, por el contrario, hemos considerado sólo su diferir mismo. A y B difieren; pero de una manera más originaria que como diferiría, pongamos por caso, el rojo de un sonido. Ambos difieren mutuamente $y$, por diferir, se nos dan; pero el rojo debe haberse ya dado para diferir de un sonido, esto es, debe ser ya diferente de un color, para ser diferente de otra cosa. Podríamos introducir una imagen y decir que, en el campo del conocimiento directo, algunos cognoscibles difieren originariamente entre sí y son, por ello, miembros de una categoría, v.gr., la categoría de color o de sonido, etcétera, si así queremos llamarlas, y que su estancia en ese campo de conocimiento no requiere la estancia de miembros de otra categoría. Es obvio que se pueden conocer sonidos únicamente y no colores, $y$ a la inversa.

A reserva de insistir en el tema, queda examinar, por último, la posibilidad de que tres o más colores sean similares, así sea para desechar la suposición de que el número de los mismos fuera, en gran medida, fundamento de su similitud. Sean A, B y C tres colores, sin repetición, que difieren minimamente entre sí, de suerte que el tercero no sea el mismo que el segundo o el primero. (Contar colores ofrece, en oca. siones, dificultades. En un sentido podrían ser tres, tanto como dos en otro. Observación no tan trivial, como se verá más adelante.) Ahora bien, tampoco, a primera vista, parecen ser similares estos tres colores. Para convencernos de ello basta considerar el caso - reducible, en último término, al anterior, donde entraban sólo dos colores- en que $\mathrm{A}$ difiera mínimamente de $\mathrm{B}, \mathrm{B}$ de $\mathrm{C}$ y $\mathrm{C}$, a su vez, de $\mathrm{A}$. Su realidad ha sido aceptada, implícita o explícitamente, repetidas veces, en la literatura psicológica, aunque basta simplemente establecer su mera posibilidad para que nos per-

4 Locke, Essay, III, 4, 6. 
catemos de que no es la única. La más interesante, sin duda, es la de que $A$ difiera mínimamente de $B, B$ de $C$; pero $C$ difiera no mínimamente de $A$. Lo decisivo aquí es la presencia de $\mathrm{B}$; pues únicamente si elimináramos a $\mathrm{B}$, en cuanto color dado o conocido, diferirían A y $\mathrm{C}$ mínimamente. La manera en que $A$ y $B$ se nos dan y difieren depende de $B$. Con esto tenemos ya un orden. Una línea, donde cabe agregar un color más que difiera mínimamente de $\mathrm{C}$, pero no así de $B$ y de $A$, o un color que difiera mínimamente de $A$, pero difiera no mínimamente de $\mathrm{B}$ y de $\mathrm{C}$, y así en adelante. Nuestra tesis es que tal orden es un ejemplo de similitud y, más precisamente, de similitud entre colores. Los colores $A$ y $B$ son similares si $C$ es más similar a $B$ que a $A$, por diferir mínimamente de $B$ y no mínimamente de $A$, en tanto que A sería tan similar a $B$ como $B$ a $A$, etc. Aun expuesta de esta manera intuitiva, sin más recurso que el poder expresivo del lenguaje natural, puede verse que dicha similitud es una relación construida sobre la base de una actitud teórica que destaca una posibilidad y excluye cualquier otra; pero que se funda en algo real, en una manera de dársenos, en el conocimiento directo, tres colores por lo menos.

Una de las consecuencias de lo anterior es que nos veremos en dificultades para encontrar un límite en el ordenamiento de colores similares a un primero dado, a no ser que lo fijemos arbitrariamente. Por ejemplo, en las encuestas llevadas a cabo por Berlin y Kay, ${ }^{\circ}$ con hablantes nativos de veinte leguas naturales distintas, acerca del uso de los términos básicos de color, se pidió a los informantes que señalaran en la carta de colores Munsell, todas aquellas muestras que, bajo cualesquiera condiciones, llamarían con el término de color básico X. En repetidas pruebas, aun con un mismo informante, en circunstancias normalizadas, no se logró establecer que, en lengua alguna, la extensión de un término de color cualquiera se mantenga dentro de límites seguros.

5 Berlin y Kay, Basic Color Terms, University of California Press, 1969, pp. 5-7. 
El resultado era de preverse. Una vez señalado el color escogido como referente del término, sólo arbitrariamente se podía limitar la totalidad de muestras de color similares al escogido. Esta extensión de un término de color, constituida por un color elegido a manera de paradigma y por los colores similares a él, tiene necesariamente que ser borrosa, porque sus límites son confusos y sólo determinables arbitrariamente. Lo importante es señalar que, pese a ello, también puede haber extensiones de colores cuyos límites estén absolutamente fijados y no dependan del arbitrio. Cuáles sean sus requisitos lo veremos más adelante; por lo pronto detengámonos un poco en este color paradigma antes aludido.

De manera muy imprecisa hemos hablado de un color elegido como referente del término $\mathrm{X}$, como si no pudiera ser más que uno y su desaparición dejara sin referente al término, poniéndonos en la situación de decir: no lo encuentro entre las muestras; no puedo señalarlo. Por otro lado, aun si son elegibles más de uno, tampoco estamos en una libertad de condiciones como para elegir cualesquiera colores de los de la cadena indefinida de similares. Tienen que ser los mejores ejemplos de los que hemos aprendido a designar con tal término de color. No será difícil determinar cuáles sean; puesto que no entrará en juego la similitud entre ellos, aunque sí intervendrá un factor nuevo: su identidad con los colores que hemos aprendido a designar con el término $\mathrm{X}$.

A este respecto es muy ilustrativo otro de los resultados de las investigaciones de Berlin y Kay. ${ }^{\circ}$ Interrogados los mismos informantes acerca de cuál o cuáles de esas muestras eran los mejores ejemplos de $\mathrm{X}$, los resultados fueron sorprendentemente uniformes, no sólo individual y colectivamente, dentro de una misma lengua, sino en las veinte lenguas estudiadas. En casi todos los casos, los colores señalados fueron los mismos, en correspondencia con grupos de términos intertraducibles. Dejando a un lado las im-

- Berlin y Kay, op. cit., pp. 7 y 13-14. 
plicaciones que esto pudiera tener por lo que toca a la tesis de los universales lingüísticos materiales, cabe preguntarse por la razón de dichos resultados. Estos llamados focos de color parecen haberse constituido en torno a un paradigma y estar integrados, primero, por muestras no similares a él, pero del cual todas difieren mínimamente, y segundo, aunque no siempre, por el color paradigma mismo. Cualquiera de ellos sin más, podrá ser el referente de un mismo término de color.

Ahora bien, cuál de esos colores funcione como paradigma es arbitrario. Si, además, coincide con el referente de un término de color, el referente podrá ser cualquiera de aquellos colores que difieran todos mínimamente entre sí, pero una vez elegido alguno, en una ocasión, continuará siéndolo en las demás. Dicho de otra manera, una vez elegido tal paradigma o, en su defecto, otro, ese color y todos los que sean idénticos a él funcionarán como el mismo referente de X. Estas afirmaciones requieren ciertamente ser elucidadas y desarrolladas, ya que nos evitarán admitir la problemática tesis de que un término de color conlleve una intensión que funcione, en toda ocasión, como regla para elegir su posible referente.

En este sentido, un paradigma tendría que ser un color más entre otros. En un primer momento, todos aquellos colores frente a los que funciona como paradigma son sus mejores "copias". La razón del enlace entre uno y otras se funda sólo en la mínima diferencia que media entre ellos; la mínima - repitamos - que lleve a conocerlos directamente como colores distintos, sin intermediarios. Un paradigma podría representarse como un punto, un foco, en un espacio de tres dimensiones, en torno del cual se situarían sus posibles mejores copias a una mínima distancia, difiriendo cada una mínimamente de otra, a la vez que del paradigma. Pero, de acuerdo con lo dicho anteriormente, también habrá copias que no sean calificables como las mejores, por cuanto difieran no mínimamente de su paradigma. A partir del foco $A$, 
pasaríamos, en un orden lineal, a su mejor copia $B$, y de aquí a $C$, que difiere mínimamente de $B$, pero no de $A$, luego llegaríamos a $\mathrm{D}, \mathrm{y}$ así en adelante. $\mathrm{C}$ y $\mathrm{D}$ serían similares a su paradigma, como lo sería aquí $\mathrm{B}$, en cuanto miembro de este ordenamiento.

La noción de paradigma expuesta hasta ahora parece ser incompleta, si no es que inadecuada; pues según lo dicho, el color paradigma cumpliría meramente su función respecto a colores diferentes de él, pero no puede excluirse en modo alguno la posibilidad de que la cumpla también con respecto a colores iguales a él, dado que cabe reconocerlos como el mismo color. Si es así, ¿qué sentido tendría hablar de colores idénticos? ¿Sería uno y no varios, o serían meramente similares en un grado último?

La función del paradigma se ha presentado como la de establecer, en un ámbito de colores diferentes, un criterio para determinar cuáles de ellos son o no sus ejemplares o copias, y en qué medida lo son. Función muy importante y que no es obstáculo para que, a la vez, cumpla otro papel nuestro color paradigma. En efecto, cuando decimos, v.gr., que una superficie, en tal momento, tiene o presenta un único color, damos a entender que una parte de ella tiene el mismo color que otra; tal parte es igual en color, en rojo - digamos- que otra. En este caso, el color a que se refiere el término 'rojo' funciona como paradigma respecto a la identidad de los colores de dos partes de una superficie. Es decir, funciona como criterio de identidad en este contexto, y no como criterio de ejemplaridad. Lo mismo sucedería si se tratara de dos superficies diferentes. Tendrán el mismo color si, ante la vista o en el recuerdo, no son distinguibles en cuanto - digamos- rojas, en un momento dado, aunque sean bien distinguibles en cuanto superficies.

Como puede verse, es aquí el rojo de una superficie, o de una parte de ella, el que funciona, en estos casos, como paradigma del color de la otra superficie o de la otra parte. Paradigma ciertamente, pues variando los ejemplos podrá 
verse que, de acuerdo con él, cualquier superficie, en cualquier tiempo y en cualquier mundo, real o posible, tendrá el mismo color rojo que el de la superficie que recuerdo o tengo ante la vista. En este sentido habría que reformular la tesis de Putnam, tan apretadamente expresada: "yellow' as $a$ thing predicate is indexical"'; puesto que el término 'rojo', en los casos señalados, está tomado, en efecto, como predicado de superficies y conlleva un componente indéxico: mienta una propiedad que siempre es la misma que ésta, o, mejor dicho, que la de esto, en el mundo en que la conozco directamente junto con su portador.

Claro está que si 'rojo', qua predicado, es indéxico, ello excluye que miente, cada vez que se le usa, una única e indivisible propiedad, suerte de universal sensible del que participarían esta superficie y aquella. No; lo que mienta en cada caso es la propiedad de esto y de nada más; la propiedad de aquello y de nada más; pero respecto a la cual esto y aquello no se distinguen. Con lo cual se nos presenta de nuevo el problema ya señalado anteriormente. ¿Se trata de una o de varias propiedades? ¿Pueden varias propiedades ser idénticas sin ser una solamente? Si son varias ¿̇erá su identidad una especie de similitud en grado máximo? Husserl, para citar un ejemplo, trató de dar una respuesta común a estas interrogantes. Sus tesis son iluminadoras, en la medida que señalan los peligros que acechan a toda posible respuesta. Para él, la igualdad es el límite a que tienden los grados de similitud. "Die... Ähnlichkeit kann verschiedene Grade haben mit dem Limes vollkommenster Verwandschaft, der abstandslosen Gleichheit." 8 Pero, dado que concibe la similitud en términos de una coincidencia parcial, en la que una parte de $A$ recubre una de $B \longrightarrow$ en lo que son iguales-, a la vez que otra parte de $A$ es diferente de otra de $B$-en lo que difieren-, puede llevar adelante esta imagen de

7 H. Putnam, "The Meaning of 'Meaning", en K. Gunderson (ed.) Language, Mind, and Knowledge, University of Minnesota Press, 1975, p. 187.

8 E. Husserl, Erfahrung und Urteil, Classen \& Goverts, 1948, p. 77. 
eclipse parcial de B por A hasta convertirla en la de un eclipse total, en que $A$ recubre por completo a $B$, cuando son idénticas, sin que se deje de conocer a ninguna, pues uno de los términos de la igualdad se transparenta en el otro. Así - nos dice- "das Verdeckte durch das Verdeckende voll hindurch gesehen wird". ${ }^{8} \mathrm{La}$ dualidad de A y B se ha transformado en una unidad donde se preserva la dualidad. Materialmente no hay separación: uno no está fuera del otro; sino, más bien, uno está dentro del otro y, en tal medida, son dos a la par que uno. Dos; en que conocer al primero es conocer al segundo por transparencia.

Debemos evitar ser cautivados por esta imagen. Dos colores similares no lo son por recubrirse parcialmente, ni su identidad los pone uno dentro de otro. Sin embargo, el propósito de Husserl merece retomarse. Hay que procurar guardar la diversidad e indivdualidad de los colores que se presentan como el mismo en esta y aquella superficies, sin que por eso no sean el mismo, sino sólo similares, $y$, por ende, diferentes.

El aire paradójico de este proyecto se desvanecerá si repensamos la cuestión dentro de los límites en que la hemos mostrado. Hay que recorrer de nuevo parte del camino que nos ha conducido hasta aquí, pero con nuevos ojos: manteniendo la mirada en la individuación de los colores implícitamente establecida.

Solamente se nos da un color si no se confunde con otro, si se distingue de otro. Donde otro es justamente un color determinado que, a su vez, es diferente del primero, pero no es un sonido, por ejemplo. Obviamente, si tenemos un rojo, no hay el riesgo de confundirlo con un sonido. En este sentido es como afirmábamos que rojo pertenece a la categoría de color, a la que pertenece todo con lo que no debe confundirse, o todo de lo que se distingue, para ser rojo. Un rojo se distingue también de un sonido, pero notemos una vez más que se diferencia de él de otra manera, como ya

- E. Husserl, op. cit., p. 225. 
dado, en cuanto diferente ya de otro color determinado, dado que es imposible confundirlo con tal sonido.

Si consideramos, en forma igualmente intuitiva, en qué pueda consistir la individuación de cada miembro de una categoría, en especial de la categoría de color, para circunscribirnos a nuestro tema, parece imponerse de modo natural la hipótesis de que la individuación de un miembro consiste precisamente en ser diferente de otros miembros de esa categoría. ${ }^{10}$ Tendríamos un criterio de individuación relativo a una categoría, que daría razón, para comenzar, del caso en que dos superficies son idénticas en color, en rojo, por ejemplo, y son diferentes entre sí.

Llevemos a cabo un Gedankenexperiment más. Supongamos que tenemos una superficie de un solo color y que la rompemos en dos partes. Ahora, una superficie $A$ se diferencia de otra superficie B; una no se confunde con la otra ni hay riesgo de confundirla con un sonido. El color que presentaba originalmente no se ha refugiado en $A$ o en $B$, al dársenos éstas, ni se ha transformado en un universal compartido por esta y esa superficies. Tampoco tenemos ahora dos colores similares, por mínima que fuera la diferencia que los separara; suficiente, sin embargo, para distinguirlos al conocerlos directamente. El color de $\mathrm{A}$ y el color de B, por lo que toca a su categoría, siguen siendo un individuo, ya que no difieren entre sí.

Lo que sucede es que la individuación de la primera y la segunda superficie no coincide con la individuación de ese color. En un nivel tenemos dos individuos (las superficies rojas A y B); en otro nivel un solo individuo (un color: rojo). Igualmente podrían invertirse estos niveles; podríamos tener un solo individuo (una superficie) y dos individuos (rojo y verde), como ocurre cuando una misma superficie presenta esos dos colores. $Y$, así como una superficie no deja de ser un individuo por exhibir dos colores, un

10 Nótese que la diferencia entre colores no es una relación que se establezca entre colores ya dados, sino que es el darse mismo de ellos. 
color no deja de ser un individuo por estar en dos superficies.

De conformidad con lo anterior, al establecer la identidad de dos colores lo que tenemos a la vista es un solo individuo, tal o cual color, en un nivel de individuación, y, además, dos colores, puesto que, en el nivel de individuación de las superficies coloreadas $\mathrm{A}$ y $\mathrm{B}$, uno es el color de este individuo $\mathrm{y}$ otro es el color de este otro individuo. Como propiedades de individuos son dos; pero, como tal color y no otro, se trata de un único individuo.

Quedan así respondidas las preguntas acerca de las conflictivas unidad y diversidad de la identidad de los colores. Respecto a ellos no cabe decir que sean el mismo F, donde $F$ fuera siempre un término general. Se ha visto que, en una interpretación de F, para la que la expresión "el mismo" sirviera como criterio de identidad - con lo que tendríamos, según Geach, un término "substantival"-, este término podría ser no general sino individual, como cuando decimos: "este color es el mismo rojo que este otro". Igualmente queda aclarada la confusión, en este campo específico, entre similitud e identidad.

Hablar de colores - como puede verse- resulta viejo y nuevo a la vez, pues exige conjugar los temas clásicos de la filosofía con los actuales. De tal suerte, otro resultado de las hipótesis anteriores sería la posible tesis de que un predicado de color sea indéxico o deíctico, ya que en cada oportunidad co-mencionará, por así decirlo, un color que, en cuanto propiedad, siempre será la misma que ésta, la de este individuo, la tomada como paradigma, de modo que no habrá un mundo posible en que ese color no sea el mismo que éste, conocido y elegido como referente de mi término de color en un mundo real.

Sin embargo, la cuestión acerca de si un enunciado, del cual formara parte un predicado de color, pudiera ser irrevisable, epistemológicamente necesario, a la vez que metafísicamente necesario - válido en todos los mundos posibles, 
en el sentido de Kripke - y todo predicado de color fuera una suerte de designador rígido, es algo que, por el momento, no estamos en situación de analizar. Esperemos que, en un futuro, otras investigaciones arrojarán luz sobre la legitimidad misma de esta cuestión y sus insospechados alcances. 
The aim of the paper is to give an account of the similarity of colors as well as of their identity and individuation, even if the author's comments will get their full share of meaning only within the horizon of a broader theory which so far is incomplete.

In what follows we will take colors as they are given to us when we are directly acquainted with them, i.e., we will leave aside any consideration of colors in terms of physical or scientific theories and our way of dealing with their shades of color will be to take any two different ones as being two different colors. On the other hand, the problems we are confronted with will spring if these colors are given to us as simples.

Let us start by assuming that we certainly know two different colors, $A$ and $B$, which are such that their difference is the least one sufficing for them to be two colors given to us and not just one. Now, it can be asked whether these two colors are similar. What we do have is two different colors, even if they only differ minimally. To give an account of the two colors being similar one might be tempted to appeal to a traditional answer: they must have something common to both and something else, besides, which is why they are not the same but only similar. But we do not have this answer at hand since, in this case, to say that the two colors are minimally different is to say they are different altogether. But as least -it could be objected - they must have something in common for them both to be colors and not something else; e.g., they must have one and the same relation as intentional objects of vision, or be ideas intromitted by sight, as Locke understood this point, or something like this.

To the above it can be pointed out that in saying that $A$ and $B$ are colors, we have not referred to anything common to both but, as opposed to this, we have just considered their being different; $A$ and $B$ are different but in a more basic way than that in which red, say, would differ from a sound. $A$ and $B$ are mutually different and, because they do differ, they are given to us; but red has to be already given to differ from a sound, i.e., it has already to be different from a color for it to be different from something else.

Now, if we add to our list a third color, $C$, such that the three of them are again minimally different among themselves, this does not seem to help us to conclude that they are similar. Since, assuming $A$ minimally to differ from $C$ and $C$ minimally to differ from $A$, 
we have again a case that can be reduced to the former one in which we had just two colors with a minimal difference between them. But now, if we consider another possibility, one in which three colors, $A, B$, and $C$, are given to us such that $A$ differs minimally from $B$, $B$ differs minimally from $C$, but where $C$ differs not minimally from $A$, we have an order, a line which can be lenghtened by adding colors $D, E$, such that $D$ differs minimally from $C$ but not from $B$ or $A$ and $E$ differs minimally from $A$ but not from $B, C$ or $D$, etc. We claim that such an order is an example of similarity and, more precisely, of similarity among colors.

A difficulty one is faced with in taking the above line of though is that one has a problem in finding a limit, unless an arbitrary one is fixed, in the ordering of colors similar to a given one. Ex. perimental research, by Berlin and Kay, has shown that the above is certainly the case: once a color was chosen as the reference of a term, the totality of color samples similar to the one chosen could only be arbitrarily limited.

But Berlin and Kay also found that there was nothing arbitrary in the selection of the best exemplars of color $X$. These so called color focuses seem to be constituted around a paradigm and to be integrated, firstly, by exemplars not similar to, but which differ minimally from, it and, secondly, althought not always, by the paradigm itself.

Now, which one of those colors functions as a paradigm, is arbitrary. If, besides, it coincides with the referent of a color term, the referent can be whichever of those colors which, among them. selves, differ minimally; but once the referent is chosen it will be the same in any further ocassion.

A paradigm might be represented as a point, a focus, in a three dimensional space, around which would be located its possibly best copies at a minimal distance, each one of them differing minimally among themselves as well as from the paradigm. But, as has been said, there will also be copies which will not qualify as the best in virtue of their not being minimally different from this paradigm.

But now, by what has been said, the paradigm color would fulfill its function with regard to colors different from it, but the possibility of its fulfilling the same function with respect to colors which are the same as it, cannot be excluded. If this is so, what would be the sense of talking of identical colors? Would they be the same and not several or would they just be similar in an ultimate degree?

When we say, e.g., that at a certain time a surface has or shows a unique color, what we mean is that part of that surface has the same color as the other; it is the same red, say, as the other. In such a case, the color we are refering to by means of "red" is func- 
tioning as a paradigm with respect to the identity of the color of both parts of the surface. I.e. it is functioning, in this context, as an identity criterion and not as a criterion of exemplarity.

But now, what can be done with Putnam's dictum "yellow' as $a$ thing predicate is indexical'? Since, certainly, in the case we have considered "red" has been taken as a predicate about surfaces, it carries it an indexical commitment. But if "red", as a predicate, is indexical, this precludes that it names, each time it is used, a unique and indivisible property, some sort of sensible universal of which this and that surfaces would partake. No, what in each case it names is the property of this and of nothing else; the property of that and of nothing else. But with respect to which this is not distinguished from that. And now, again, we are confronted with a problem already mentioned: Are we dealing with just one or with several properties? Can several properties be identical without being just one? If several, will their identity be some sort of similarity of maximum degree?

Husserls, as an example, gave a common answer to these questions: sameness is the limit approach by degrees of similarity. Since he thinks of similarity as a spatial coincidence by means of which a part of $A$ covers a part of $B$-in that in which they are the samewhile at the same time another part of $A$ is different from another part of $B$-in that in which they differ- he can proceed with the image of a partial eclipse of $B$ by $A$ to obtain a total eclipse in which $A$ completely covers $B$-when they are identical- but with both terms of this equality being transparent to each other.

Even if this image is to be rejected, Husserl's intention is to be taken into account: the diversity and individuality of colors which present themselves as the same on this and that surfaces has to be preserved, maintaining their sameness and, at the same time, their similarity and, so, their difference.

The air of paradox within this project will settle once we consider this matter within the boundaries within which we have stated it.

What we have said is that a color is given to us only if it is not to be confused with another color, i.e., where the distinction is made within the bounds of a certain category, that of color in our example. And, again, a color is individuated if it is different from other members of the same category, i.e., from another colors. And this can give us a starting point to account for the fact that two surfaces are identical in color, in red, say, but different between themselves.

Let us suppose, now, that we break into two halves a one colored surface. Surface $A$ will be different from surface $B$. But the color of $A$ and the color of $B$, with respect to their category, are still one and the same individual, since they do not differ. 
What happens is that the individuation of surfaces is not the same as that of colors. At a certain level we do have two individuals - the two red surfaces $A$ and $B$; at another level we just have one individual -a color: red.

And now, what we do have is that red, as a property of the two surfaces $A$ and $B$, is two colors; but as being that color and no other, is just one individual color.

We have thus answered the questions about the conflictive unity and diversity of color identity. With respect to colors, it cannot be said that they are the same $F$, where $F$ would always be a general term. As has been shown, this $F$ might, sometimes, be an individual term as when we say: "This color is the same red as that one."

As another result of our former hypotheses, we might have the possible view that a color predicate be indexical or deictical since every time it will co-mention, so to say, a color which as a property always will be the same as this, the one pertaining to this individual, the one taken as a paradigm, so that there will be no possible world in which that color is not the same as this, known and chosen, in an actual world, as the referent of my color term.

[J. A. Robles] 